\title{
The content and present situation of experimental medical studies
}

\author{
Jinguo Wang ${ }^{1, a}$ and Na Wang ${ }^{2, b^{*}}$ \\ ${ }^{1}$ the First Hospital of Jilin University, Changchun 130021, China; \\ 2 the First Hospital of Jilin University, Changchun 130021, China \\ awangjinguolily@163.com, bwangna080613@163.com
}

Keywords: Experiment, medical studies, legal regulation.

\begin{abstract}
Experimental medical practice is essential to the human medical research, which brings to the human progress. But without limitation of comprehensive and standardized legal regulation, the rights and interests of patients will be greatly violated and invaded. In this study, suggestions are provided about subjects protection mechanism, adverse reactions rescue mechanism, related law construction, compulsory insurance to improve the protective system of experimental medical practice.
\end{abstract}

\section{Introduction}

Now there are a lot of illegal society experimental medical behavior. Because there is no legal regulation to regulate this behavior, resulting many realistic social issues and a public debate. Mainly the human body is in the process of the experimental study on the researchers told not fully, the subjects' rights and interests can not get the actual security, damage when subjects were difficult to seek the judicial relief process.

\section{Text}

Experimental medical treatment according to the test object classification are divided into: medical clinical trials, new technology and new drug clinical trials.

Generalized medical technology is applied to health care and medical services system specific knowledge of the system, including medical methods, procedures and related organization system.Belong to the combination of hardware and software systems. Special medical technology refers to the medical institutions and medical personnel for the purpose of diagnosis and treatment of disease, the disease make judgments, to eliminate the disease, relieve the illness, pain relief and improve function, prolong life, help patients to restore health diagnosis, treatment measures and methods.

First, refers to the safety, efficacy precise, involves certain ethical issues or the risk is higher, the health administrative department shall be controlled management of medical technology. Third, including major ethical issues, high risk and safety. Efficacy still need to the specification of clinical trials research further verification. You need to use scarce resources: the ministry of health of other need special management of medical technology. This paper mainly relates to the third class medical technology, the so-called medical new technology [1].

Medical technology is widely used in clinic, is conducive to maintaining human life, to promote the health of human beings, is helpful to improve the ability of prevention and treatment of diseases of the human race. To improve the medical staff of the science level and promote human knowledge popularization of modern medicine;Promote the sustainable development of modern medical science. At the same time, we should also see, the medical application of new technology also brings a series of ethical and legal problems.Representative and the well-known medical new technology including: artificial reproductive technology, organ transplantation, gene technology, and video diagnostic technology, laser technology, stereotactic technology, all belong to the category of new technology. Its scope is widespread, includes the following characteristics.

The improvement of material life to promote people's further attention to health. The strong 
requirement of public health from the objective to promote the health of the new technology research and development, innovation constantly. At the same time, the public health needs of the new medical technology to speed up the industrialization process of market demand. Because of the medical technology industry has the characteristics of high returns, priority to the development of medical technology can promote the development of national economy, the high returns constitute the power source of accelerating the industrialization process [2]. Inducing factors of accelerating and new medical technology industrialization process is the fundamental interests of the people to the survival and health, the medical technology industry became the various countries' demand structure and economic structure continuity and be full of vitality of the emerging industry.

Modern medicine, new technology applications, organ transplantation, artificial reproductive technology, gene technology, modified new medical technology, such as the risks of the unknown, the types of technology is the most common and controversial. We know from the concept of medical new technology, new technology means that the unknown and explore. To the progress of medical science, in the process of the whole human trials using double-blind, placebo experiment, the control group, randomised trials test method on the subjects of the test of the new technology.Because for the unknown process, efficacy, safety, the subjects easily cause harm to the body and mind.Medical technology involves multi-disciplinary comprehensive application, may be or has been involved in many current difficult to solve the problem such as medicine, law, society. Such as: the application of artificial reproduction technology, because it changes the nature of the human society has long reproductive reproduction behavior, produce the artificial fertilization, in vitro fertilization methods of new technology. This new technology has brought more tyres, embryo business problems. Of human society has always challenged the family, traditional social ethics [3].

Medical research and development of new technology and innovation is a necessary stage of the medical technology progress, but in the process of assessment, human limited by knowledge and may impede the progress of medical technology.Research and development and application of new technology to the human medical history continuously injected fresh blood, and the generation of this kind of opportunity is always accompanied by risk accompanying. When we control the risk also means may lose opportunities. Such as: early bone marrow transplant technology development, the effectiveness of chemotherapy and bone marrow transplantation is limited, in the late clinical trials of good effects is a doctor by repeating to make these technologies more safe and effective. But in the early days of technological inventions, if involved in technology access, assessment mechanism, will bring what kind of results? Whether because the regulation to restrict the development of new technology, is also a combination of contradictions. So, in the protection of the rights of patients at the same time, between the control risk and promote the development of medical technology to obtain the certain balance.

Our country "pharmaceutical administration law" the one hundred and second regulation: the drug, is used in the prevention, treatment and diagnosis of disease, purposefully regulate physiological function and regulation have indications or functions of indications, usage and dosage of the material, including traditional Chinese medicine, Chinese medicine yinpian, proprietary Chinese medicine, chemical API is extremely preparations, antibiotics, biochemical drugs, radioactive pharmaceuticals, serum, vaccines, blood products, diagnostic drugs and so on. Drug clinical trial refers to the human body (patients or healthy volunteers) in clinical experimental studies about the effect of a series of new drugs, to confirm or reveal experimental drug efficacy and adverse reactions, the purpose is to determine the curative effect and security of the test drug. With the development of medical science and technology in China, there is growing recognition that clinical trials of drugs, clinical trial work towards scientific, procedural and standardized: "treatment, is a doctor with has proved to be effective therapy, to treat the patient [4]. Patients are beneficiaries or being cured, or relieve symptoms. The drug clinical trials, the result is uncertain. After animal testing, new drug must be entered into the phase of clinical trial. For animals and people on the effectiveness of new drugs and toxic reaction is different, so the ongoing drug clinical trials, direct purpose is not to make directly benefit subjects, but in order to understand new drugs on the human body of pharmacological effects, 
adverse reaction and adaptability, the scientific conclusion. In order to sale of new drugs. Because of its curative effect on the human body not sure, so treat purpose on the dog, and has certain risks.

Through above to know, the process is carried out independently by the applicant applied research stage, involved in the drug post-marketing surveillance. Because of facing the global many lawsuits, Merck face about tens of billions of litigation costs. Happen this situation, the United States food and drug administration also embarrassing, accommodative.In our country, because of medical compensation system is in its infancy, many of the medical tort caused by medical damage compensation mechanism in a state of absence. Involved in drug clinical trials, because of its is unknown, the risk coefficient of high industry, in the process of test, such as the subjects was hurt, can't find the corresponding medical compensation, insurance and related economic compensation mechanism.

Along with our country on drug clinical trial related management regulations of constantly develop and perfect, new drug research and development process become more complicated and tedious, the higher the costs.Pharmaceutical companies in the strict management, competitive environment to survive and development, it is necessary to shorten the time required for new drug research and development, to control costs and reduce risk. Also fostered "Contract Research Organization (Contract Research Organization, CRO), its role is to develop clinical test plan, test plan and the contract of cooperation, the importance of the work content for the whole link is an indispensable part of the clinical trials [5]. At the same time, such an organization, the level of work and management coordination ability is directly related to the quality and efficiency of drug clinical trials.But in the CRO in our country is still in its infancy, for its own business scope and business model is not clear, lack of practical experience, the efficiency is not high, the lack of internationalization of professional talents.But with the development of medical science and the constant improvement of China's relevant laws and regulations, the defects of CRO itself make its development into a bottleneck, from the objective to promote it to take measures to improve their professional level.

The development and innovation of medical equipment has entered the new stage, rapid development, but because of the medical equipment emerge in endlessly, treatment is applied to the patients also countless problems caused.Medical apparatus and instruments of new products, it is to point to the domestic market has not yet appeared, or security, effectiveness and product mechanism has not been approved by the domestic new varieties.In order to increase the accuracy of the results, the medical instrument clinical trial subjects should be selected to ensure homogeneity.

Post-market medical devices are widely used in different individuals, some defects, only after a long period, a large number of clinical use of new products can be found.So, listed on the test and the actual use situation, comparing to the experiment defects found in the process of new product has limitations.Because of the particularity of medical apparatus and instruments, enter clinical trials, from the design, production, market, there are some risks.By above knowable, at present a lot of new medical instruments by continual research and development, the market sales.

In order to guarantee the life and health of the patients, medical apparatus and instruments related laws and regulations in our country mainly puts forward regulatory requirements on equipment listed before but for the new instruments listed after the follow-up supervision is seldom involved.For this on the one hand, in Europe and the United States had more strict requirements.Such as: the United States for medical new equipment of dynamic regulation is mainly by the food and drug administration (FDA) by medical device reporting system, medical equipment, medical equipment tracking system to implement recall system.In order to promote the development of new medical equipment industry in our country without hurting the rights and interests of patients.

By China's regulations on the supervision and administration of medical devices for the classification of medical apparatus and instruments as we know, belong to the second, three kinds of products of medical instruments, in promoting the development of medicine at the same time, their use has a greater risk to the human body. Has not been seen in the domestic market due to its security, effectiveness and product recognition mechanism have not been home, in the clinical results of the application of the unknown. Of this kind of products at the same time, such as using the physician of 
deep understanding of the product, skill is not enough skilled, more increased the risk of using coefficient.Therefore, the use of physician skill of this new equipment.

The rules for the implementation of the at that time the people reasonable specification provides legal protection. On December 1, 2001 implementation of the pharmaceutical administration law of the People's Republic of China, indicates the drug into the 21 century in China, the legal regulation of the progress, for after a large number of new drug research and development. On September 15, 2002 of the "drugadministration law of the People's Republic of China regulations on the implementation of the fifth chapter 28, 29, 30, 34, 35 content are for the new drug, drug clinical trials of the drug administration law. The earliest laws and regulations of the subjects right of informed consent is effective as of September 1, 1994 of the implementing rules for the regulation of medical institutions. The author believed that the drug clinical trial has specific regulation of laws and regulations is really on September 1, 2003 of the "quality control standard for clinical trials" (hereinafter referred to as the "specification")." Specification" for the purpose of the specification is to ensure that drug clinical trial process, the result scientific and reliable, protection of rights and interests of the subjects, and guarantee its safety. Which mainly by way of establishing and perfecting the ethics committee for the protection of the rights of the subjects, in line with the international standards of ethical review. Clinical trials at the same time, including the preparation and the necessary conditions, the content of the test solution, the researcher's responsibilities, the sponsor of the inspector's duties, record and report, data management and statistical analysis, the experiment with the drugs management, quality assurance, multicenter trial, etc. Relative to other specific regulation, the quality control standard for clinical trials is by far the most comprehensive in terms of drug clinical trials, detailed specification, has representative significance.

\section{Summary}

Over the years, both drug clinical trials and medical device clinical trials, the subjects' rights and interests problem have always been the focus of attention of the masses. Because experimental medical behavior laws in our country is in the primary stage of development, subjects were damaged in the process of experiment can't find the unified regulations for protection is a frequent phenomenon. Provisions in the medical device clinical trials in the second chapter, mainly stated the terms to the guarantee of rights and interests of the subjects, including cost, the content of the subjects right of informed consent, privacy and highlight the knowledge.

\section{References}

[1] Laurent Adumeau. Regioselective Grafting of Antibodies Fragments on Multimodal Nanoprobes for Early Stage Atherosclerosis Disease Diagnosis [A].

[2] Wei Qin,Yong Zhang,Longyin Zhang,Xianxian Wu,Ning Du,Yingying Hu,Xiaoguang Li,Nannan Shen. MicroRNA-26a Prevents Endothelial Cell Apoptosis by Directly Targeting TRPC6 in the Setting of Atherosclerosis[A].

[3] Yingmei Feng,Dong Zhao,Catherine Verfaillie. Role of High-Density Lipoproteins in the Regulation of Hematopoietic Stem/Progenitor Cells in Atherosclerosis [A].

[4] Annette Graham,Janice Taylor,Anne-Marie Allen. Mitochondrial Function and Regulation of Macrophage Cholesterol Homeostasis [A].

[5] Ryouta Maeba. Serum Choline Plasnialogens,Particularly Those with Oleic Acid in Sn-2,are Reliable Biomarker for Atherosclerosis [A]. 